\title{
DESEMPENHO DE MOTOR DIESEL QUATRO TEMPOS ALIMENTADO COM BIODIESEL DE ÓLEO DE SOJA (B 100)
}

\author{
Performance of four stroke diesel cycle engine supplied with soybean oil biodiesel (B 100)
}

\author{
Carlos Eduardo Silva Volpato 1 , Alexon do Prado Conde ${ }^{2}$, Jackson Antonio Barbosa ${ }^{3}$, Nilson Salvador ${ }^{4}$
}

\begin{abstract}
RESUMO
Objetivou-se, neste trabalho, avaliar o desempenho de um motor de ciclo diesel quatro tempos e quatro cilindros utilizando biodiesel de óleo de soja (B100), em comparação ao óleo diesel. Foram analisados os parâmetros: potência efetiva e reduzida, torque, consumo específico e energético de combustível, eficiência termomecânica e volumétrica. Foi instalado um ensaio com delineamento inteiramente casualizado (DIC) em esquema fatorial, realizada análise de variância e aplicado teste de Tukey, a 5\%. Foram pesquisados cinco níveis de rotação em quatro repetições $(650,570,490,410,320$ e $240 \mathrm{rpm})$. O motor alimentado com biodiesel apresentou torque e potência reduzida um pouco menor que quando alimentado com óleo diesel fóssil, entretanto, os consumos especifico e horário, apresentaram resultados mais satisfatórios que o diesel fóssil.
\end{abstract}

Termos para indexação: Combustível alternativo, eficiência energética, motor de combustão.

\begin{abstract}
The aim of this work was to compare the performance of a four stroke diesel cycle engine and a four cylinder using biodiesel made from soy oil (B100), in comparison with the diesel oil. The parameters analyzed were: effective power and reduced power, torque, specific and energetic consumption of fuel, thermal-mechanics and volumetric efficiency. An entirely randomized experiment design was installed (DIC) in a factorial structure, the analysis of variance was carried out and the Tukey test was applied at the level of 5\%. Five rotation levels were researched in four replications $(650,570,490,410,320$, and $240 \mathrm{rpm})$. The engine fed with biodiesel presented torque and reduced power a little lower than the engine fed with fossil diesel. However, specific and hourly consumptions presented more satisfactory results.
\end{abstract}

Index terms: Alternative fuel, energy efficiency, combustion engine.

(Recebido em 4 de outubro de 2007 e aprovado em 3 de junho de 2008)

\section{INTRODUÇÃO}

O biodiesel é um combustível renovável derivado de óleos vegetais, como girassol, mamona, soja, babaçu e demais oleaginosas ou de gorduras animais, usado em motores de ciclo diesel em qualquer proporção com o diesel mineral ou puro. É produzido por meio de processos químicos, normalmente por transesterificação, na qual é removida a glicerina. Tal como o álcool está para a substituição da gasolina nos motores de ciclo Otto, o biodiesel substitui o óleo diesel nos motores de ciclo diesel, com a vantagem de não requererem adaptações mecânicas. Enquanto o uso de outros combustíveis limpos, como o gás natural ou biogás e o álcool etílico, requerem adaptação, a combustão de biodiesel pode dispensá-la, configurando-se em uma alternativa técnica capaz de atender a toda a frota já existente movida a óleo diesel, além de apresentar alto rendimento energético.
Conforme Lei $\mathrm{n}^{\circ}$. 9478/97, biocombustível é o combustível derivado de biomassa renovável, para uso em motores a combustão interna ou conforme regulamento, para outro tipo de geração de energia, que possa substituir, parcial ou totalmente, combustíveis de origem fóssil.

A produção de biocombustível, a partir de óleos vegetais brutos, tem sido alvo de diversos estudos nas últimas décadas. No Brasil, a instituição do Programa Nacional de Óleos Vegetais (OVEG, 1985) permitiu a realização de testes com óleos vegetais de composição química e grau de insaturação variados. Os principais óleos testados nessa investigação foram os derivados de macaúba, pinhão-manso, indaiá, buriti, pequi, mamona, soja, babaçu, cotieira, tinguí e pupunha.

Segundo Costa Neto et al. (2000), a avaliação da qualidade carburante de óleos vegetais requer a determinação analítica de, principalmente, seu poder

\footnotetext{
'Doutor em Engenharia Agrícola - Departamento de Engenharia/DEG - Universidade Federal de Lavras/UFLA - Cx. P. 3037 - $37200-000$ - Lavras, MG volpato@ufla.br

${ }^{2}$ Mestre em Engenharia Agrícola - Centrais Elétricas de Minas Gerais/CEMIG - Rua São Paulo, 164 - Centro - $37002-110$ - Varginha, MG alconde@cemig.com.br

${ }^{3}$ Doutor em Engenharia Agrícola - Departamento de Engenharia/DEG - Universidade Federal de Lavras/UFLA - Cx. P. 3037 - $37200-000$ - Lavras, MG jackson barbosa@hotmail.com

${ }^{4}$ Doutor em Engenharia Agrícola - Departamento de Engenharia/DEG - Universidade Federal de Lavras/UFLA - Cx. P. 3037 - $37200-000$ - Lavras, MG salvador@ufla.br
} 
calorífico, índice de cetano, curva de destilação, viscosidade e ponto de névoa.

Segundo Torres et al. (2006), testes realizados com motor Agrale, modelo M-85 tipo estacionário, monocilindro, com $7,36 \mathrm{~kW}$ de potência; não foram detectadas diferenças significativas quanto à utilização de óleo diesel e de biodiesel (B100), com resultados bem próximos. O consumo específico de combustível com B100 foi, aproximadamente, 20\% maior que no óleo diesel; nas emissões de $\mathrm{CO}_{2}$ praticamente não houve alteração; porém, as emissões de $\mathrm{CO}$ foram muito maiores para potência de $4 \mathrm{~kW}$. Os resultados mostraram a possibilidade imediata da substituição do óleo diesel pelo biodiesel como combustível, nos motores estacionários de baixa potência.

Salvador (1984) realizou testes em um trator equipado com motor Agrale, modelo M-90-T, monocilindro vertical, com torque de 3,7 kgf.m a $1.800 \mathrm{rpm}$; utilizando éster metílico de Joannesia princeps Vell. a $100 \%$ e em misturas ao óleo diesel. Verificou que, em proporções de combustível B100, não houve queda de potência no motor e houve pequeno aumento no consumo de combustível na medida em que se aumentou a quantidade de éster na mistura. A eficiência térmica não foi afetada e não foram identificadas irregularidades no funcionamento do motor. Após testes, a câmara de combustão e as adjacências mostraram-se com uma fina camada de resíduos de carbono e o bico injetor mostrou-se desprovido de depósitos de carvão e sem aparência de corrosão.

Ferrari et al. (2007) utilizaram um gerador de energia elétrica e biodiesel de soja obtido por meio da transesterificação do óleo com etanol anidro na presença de catalisador alcalino $\mathrm{NaOH}$, com rendimento de $57 \%$ no processo de fabricação. Foram testadas as proporções de 5, 10, 20, 40 60, 80 e 100\% ao diesel comercial e o consumo médio, em L.h ${ }^{-1}$ de funcionamento, do equipamento mantido sob as mesmas condições de operação. Observou-se diminuição do consumo de combustível em 3,7\% para B5, 5,9\% para B10, 1,6\% para B20 e, nas demais misturas, houve um pequeno aumento no consumo.

Maziero et al. (2005) realizaram ensaios comparativos de desempenho em um motor MWM modelo 407TCA (92 kW a $3.200 \mathrm{rpm}$ ) de injeção direta, utilizando óleo diesel metropolitano e biodiesel (éster etílico de óleo de girassol, ou EEOG) como combustíveis. Com a substituição do óleo diesel por EEOG, ocorreu uma redução média de 7,6\% na potência do motor e um aumento de $9,8 \%$ no consumo de combustível. Reduções de $6,0 \%$ na potência do motor também foram encontradas por Silva et al. (2004) quando compararam o desempenho de biodiesel (B100) de óleo residual, em motor diesel MWM 4TVA.

Barbosa et al. (2008) avaliando o desempenho de um motor alimentado com óleo diesel mineral e misturas deste com biodisel nas proporções equivalentes a B2 (98\% de diesel mineral e 2\% de biodiesel), B5 (95\% de diesel mineral e $5 \%$ de biodiesel), B20 (80\% de diesel mineral e $20 \%$ de biodiesel) e B100 (100\% de biodiesel), concluíram que a potência do motor aumentava respoectivamente do B100 ao diesel mineral, entretanto, na ordem inversa, a eficiência térmica diminuia do diesel mineral para as misturas crescentes de biodiesel, sendo $4 \%$ menor para o B100. O consumo energético diminuia à medida que se aumentava a quantidade de biodiesel misturada ao diesel mineral.

\section{MATERIALE MÉTODOS}

O trabalho foi conduzido no Laboratório de Máquinas e Mecanização Agrícola do Departamento de Engenharia da Universidade Federal de Lavras. Foi instalado um experimento com delineamento inteiramente casualizado (DIC), em esquema fatorial $2 \times 6$, envolvendo dois tipos de combustível e seis níveis de rotação do motor, com quatro repetições.

Os tratamentos utilizados foram os óleos diesel comercial e biodiesel de soja (B100) em seis níveis de rotação do motor: 650, 570, 490, 410, 320 e $240 \mathrm{rpm}$, ou seja, $100,88,75 \%, 63,49$ e $37 \%$ da rotação máxima, respectivamente.

Foi feita análise de variância (ANOVA) e teste de Tukey, a 5\%, utilizando-se o programa computacional Sisvar®, nos parâmetros de torque, potência efetiva e consumo horário de combustível, em função da rotação na tomada de potência do trator (TDP).

$O$ diesel utilizado nos ensaios foi obtido na rede de abastecimento automotiva local. O biodiesel utilizado foi obtido na Usina de Biodiesel de Varginha, em base etílica. A massa específica e o índice de acidez dos combustíveis foram determinados no laboratório de Química da Faculdade de Engenharia de Varginha, em Varginha, MG. O poder calorífico superior foi determinado no laboratório de análise de óleos na Usina Térmica Igarapé, em Juatuba, MG, de propriedade da Cemig. A viscosidade dinâmica e a cinemática foram obtidas no laboratório de termodinâmica da Universidade de São Carlos - SP.

Foi utilizado um trator novo marca Massey Ferguson, modelo 275 Compacto, com motor de ciclo diesel, marca Perkins, modelo A4-4.1, com aspiração natural de 4 tempos, sistema de injeção com bomba rotativa, refrigerado a água, com 4 cilindros em linha, cilindrada 
total de $4.100 \mathrm{~cm}^{3}$, com potência nominal, segundo o fabricante, de $75 \mathrm{cv}(56 \mathrm{~kW})$, a $2.200 \mathrm{rpm}$.

Foi adotada a norma NBR ISO 1585 da Associação Brasileira de Normas Técnicas (ABNT, 1996) para determinação dos valores de torque (N.m), RPM na TDP e potência $(\mathrm{kW})$. Esses foram obtidos utilizando-se um dinamômetro, modelo NEB 200, marca AW Dynamometer (Figura 1A). Os respectivos valores de torque, potência e rotação eram acompanhados em um display digital de bancada, conforme Figura 1B.

O consumo de combustível horário do motor foi determinado por um fluxômetro marca Oval, modelo LSN41, em conjunto com seu display, o qual foi aferido para leitura em litros por hora e massa específica do fluido de 0,84 $\left(\mathrm{g} . \mathrm{cm}^{-3}\right)$. O poder calorífico inferior (PCI) $\left(\mathrm{kJ} \mathrm{kg}^{-1}\right)$ foi determinado por meio Equação 1, a partir do poder calorífico superior (PCS) $\left(\mathrm{kJ} \cdot \mathrm{kg}^{-1}\right)$, conforme Moreira (2007).

$$
P C I=P C S-3052
$$

A potência efetiva foi mostrada diretamente no display, porém, ela pode ser calculada com o torque $\mathrm{T}$, correspondente à velocidade angular pela Equação 2 (MIALHE, 1996).

$$
H_{e}=T \times N \times\left(\frac{2 \pi}{60 \times 1000}\right)
$$

em que:

$\mathrm{H}_{\mathrm{e}}=$ potência efetiva $(\mathrm{kW})$;

$\mathrm{T}=$ torque $(\mathrm{N} . \mathrm{m})$;

$\mathrm{N}=$ velocidade angular (rpm).

A potência reduzida obtida na TDP foi recalculada conforme a Equação 3 (SALVADOR, 1984).



(A)

$$
\frac{H_{r}}{H_{e}}=\frac{P_{2}}{P_{1}}\left(\frac{T_{1}}{T_{2}}\right)^{0,5}
$$

em que:

$\mathrm{H}_{\mathrm{r}}=$ potência reduzida $(\mathrm{kW})$;

$\mathrm{H}_{\mathrm{e}}=$ potência efetiva $(\mathrm{kW})$;

$\mathrm{P}_{1}=$ pressão atmosférica, por ocasião da prova $(\mathrm{mm} \mathrm{Hg})$;

$\mathrm{P}_{2}=$ pressão atmosférica padrão $(760 \mathrm{~mm} \mathrm{Hg})$;

$\mathrm{T}_{1}=$ temperatura absoluta por ocasião da prova $(\mathrm{K})$;

$\mathrm{T}_{2}=$ temperatura absoluta padrão NBR ISO 1585/1996 (298 K).

A mensuração do torque consistiu em determinar a intensidade de uma força que, atuando na extremidade de um braço, tendeu a produzir movimento de rotação. No caso de movimento rotativo contínuo, o torque é medido pelo dinamômetro (MIALHE, 1980).

Para a determinação do consumo específico, aplicou-se a Equação 4 conforme Mialhe (1996).

$$
C_{e}=\frac{C_{h}}{H_{e}}
$$

em que:

$\mathrm{C}_{\mathrm{e}}=$ consumo específico de combustível $\left[\mathrm{g} .(\mathrm{kW} . \mathrm{h})^{-1}\right.$;

$\mathrm{C}_{\mathrm{h}}=$ consumo horário de combustível $\left(\mathrm{g} \cdot \mathrm{h}^{-1}\right)$;

$\mathrm{H}_{\mathrm{e}}=$ potência efetiva $(\mathrm{kW})$.

\section{RESULTADOS E DISCUSSÃO}

As curvas para os dois combustíveis apresentaram o mesmo comportamento da curva de torque declarado na NBR ISO 1585/1996. O teste de Tukey para o torque em relação aos combustíveis (Tabela 1) indicou que o resultado

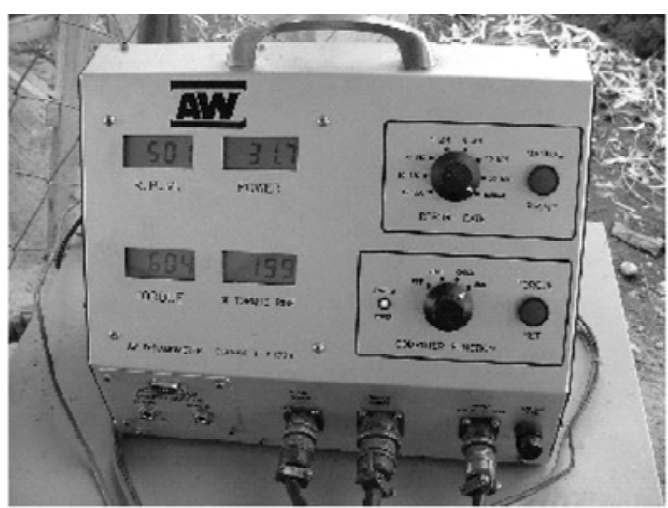

(B)

Figura 1 - Dinamômetro acoplado na TDP do trator (A) e display digital (B) 
das médias para os biocombustíveis não diferiram estatisticamente, diferindo do óleo diesel.

Tabela 1 - Médias de torque em relação aos combustíveis

\begin{tabular}{cc}
\hline Tratamentos & Médias (N.m) \\
\hline B100 soja & $589,3 \mathrm{a}$ \\
Óleo diesel & $645,2 \mathrm{~b}$ \\
\hline
\end{tabular}

Ao se analisar o efeito do torque em relação às rotações (TDP), conforme Tabela 2, observou-se que os valores médios relativos às rotações de 240 e 570 rpm não diferiram estatisticamente entre si, a $5 \%$ de probabilidade, bem como para os valores médios a 490, 410 e 320 rpm.

Tabela 2 - Médias de torque em relação aos níveis de rotação (TDP)

\begin{tabular}{cc}
\hline Tratamentos (rpm) & Médias (N.m) \\
\hline 240 & $559,25 \mathrm{a}$ \\
570 & $559,58 \mathrm{a}$ \\
490 & $626,58 \mathrm{~b}$ \\
320 & $626,92 \mathrm{~b}$ \\
410 & $648,42 \mathrm{~b}$ \\
\hline
\end{tabular}

Utilizando-se as equações polinomiais expressas nas Figuras 2 e 3 mensurou-se o torque para a rotação de trabalho, 540 rpm (TDP), ou seja, 85\% da máxima. Para óleo diesel, o torque a $540 \mathrm{rpm}$ foi de 635,3 N.m e 608,6 N.m para B100 de soja.



Figura 2 - Curva de tendência do torque para óleo diesel.

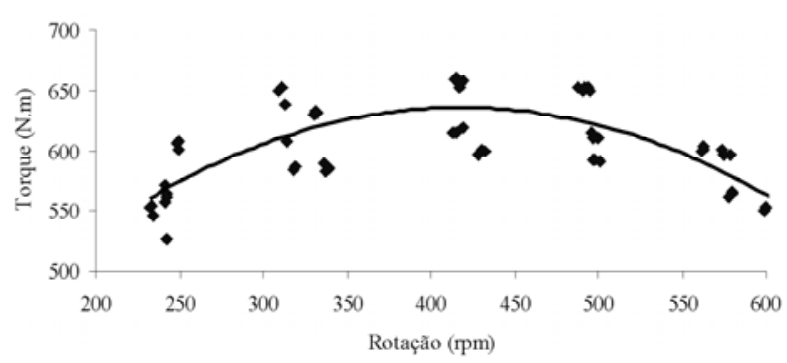

Figura 3 - Curva de tendência do torque para B100 soja.
As médias de potência efetiva em relação aos combustíveis testados estão disponíveis na Tabela 3. Pôdese observar, a 5\% de probabilidade, no teste de Tukey, que os valores da potência efetiva em relação aos combustíveis diferiram entre si e com maior valor atribuído ao óleo diesel. Verifica-se, ainda que, os valores médios para potência do B100 soja foi 5\%, menor, comparativamente ao óleo diesel, indicando que esses combustíveis apresentaram resultados tecnicamente satisfatórios.

Tabela 3 - Médias de potência efetiva em relação aos combustíveis

\begin{tabular}{cc}
\hline Tratamentos & Médias $(\mathrm{kW})$ \\
\hline B100 soja & $26,39 \mathrm{a}$ \\
Óleo diesel & $27,78 \mathrm{~b}$ \\
\hline
\end{tabular}

Ao se analisar o efeito da potência efetiva em relação às rotações na tomada de potência, conforme Tabela 4 , observou-se que os valores médios relativos às rotações de 490 e 570 rpm não diferiram estatisticamente entre si, a 5\% de probabilidade, diferentemente dos demais valores médios.

Tabela 4 - Médias da potência efetiva em relação aos níveis de rotação (TDP)

\begin{tabular}{cc}
\hline Tratamentos $(\mathrm{rpm})$ & Médias $(\mathrm{kW})$ \\
\hline 240 & $14,56 \mathrm{a}$ \\
320 & $21,13 \mathrm{~b}$ \\
410 & $27,99 \mathrm{c}$ \\
490 & $32,74 \mathrm{~d}$ \\
570 & $34,44 \mathrm{~d}$ \\
\hline
\end{tabular}

Analisando-se os resultados da potência reduzida na rotação de trabalho (540 rpm), observou-se que B100 fóssil apresentou o melhor resultado $(39,84 \mathrm{~kW})$. Na mesma rotação, obteve-se $37,53 \mathrm{~kW}$ para o B100 soja, ou seja, 6,1\%. Conforme se observa nas Figuras 4 e 5, as curvas de tendência da potência reduzida apresentaram comportamento semelhante àquelas apresentadas por Oveg (1985).

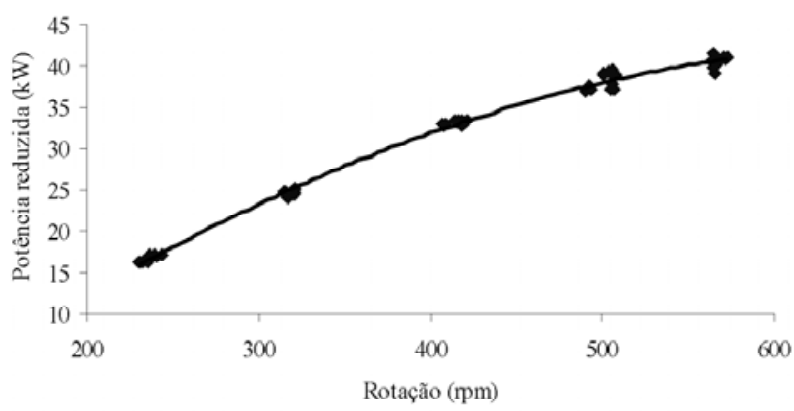

Figura 4 - Curva de tendência da potência reduzida para óleo diesel. 


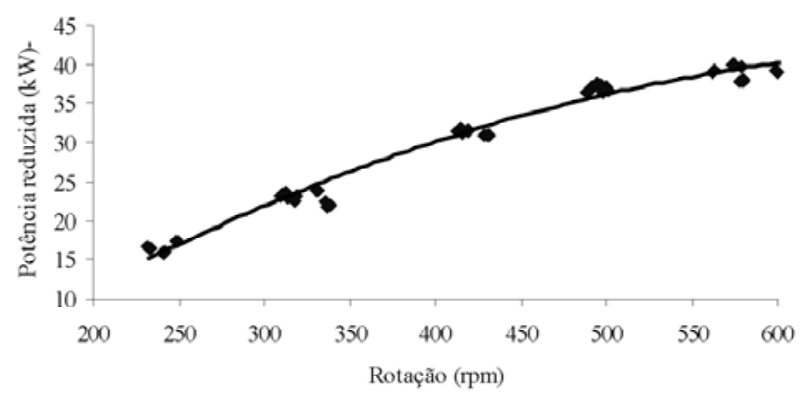

Figura 5 - Curva de tendência da potência reduzida para B100 soja.

Pelos dados da Tabela 5, verifica-se, a 5\% de probabilidade, pelo teste de Tukey, que os valores médios de consumo horário, em relação aos combustíveis testados, diferiram entre si, com maior valor atribuído ao óleo diesel. Verifica-se, ainda que os valores médios de consumo horário de B100 soja foi 17\% menor em comparação ao óleo diesel. Vale ressaltar a tendência de menor consumo para o biodiesel em motor com aspiração natural devido ao empobrecimento da mistura ocasionada por esse em relação ao óleo diesel fóssil.

Tabela 5 - Médias de consumo horário em relação dos combustíveis

\begin{tabular}{cc}
\hline Tratamentos & Médias $\left({\left.\mathrm{L} \cdot \mathrm{h}^{-1}\right)}^{-1}\right.$ \\
\hline B100 soja & $7,35 \mathrm{a}$ \\
Óleo diesel & $8,60 \mathrm{~b}$ \\
\hline
\end{tabular}

Ao se analisar o efeito do consumo horário de combustível em relação às rotações na tomada de potência, conforme Tabela 6 , observou-se que os valores médios relativos às rotações de 490 e $570 \mathrm{rpm}$ não diferiram estatisticamente entre si, a $5 \%$ de probabilidade, diferentemente dos demais valores médios.

Tabela 6 - Médias do consumo horário em relação aos níveis de rotação (TDP)

\begin{tabular}{cc}
\hline Tratamentos $(\mathrm{rpm})$ & Médias $\left(\mathrm{L} \cdot \mathrm{h}^{-1}\right)$ \\
\hline 240 & $5,75 \mathrm{a}$ \\
320 & $6,75 \mathrm{~b}$ \\
410 & $7,58 \mathrm{c}$ \\
490 & $8,58 \mathrm{~d}$ \\
570 & $8,92 \mathrm{~d}$ \\
\hline
\end{tabular}

Analisando-se os resultados de consumo específico de combustível com relação à rotação de trabalho na tomada de potência (540 rpm), observa-se que o óleo diesel apresentou maior valor médio, sendo da ordem de 257,26 g.(kW.h $)^{-1}$. O biocombustível B100 soja apresentou o melhor resultado, 224,32 g.(kW.h $)^{-1}$, o que equivale a $14,66 \%$ menos. Esse resultado é semelhante aos encontrados por Rabelo et al. (2007), quando testaram óleo de soja usado em fritura de alimentos. Nas Figuras 6 e 7 , observam-se as curvas de tendência referentes ao consumo específico dos combustíveis utilizados.

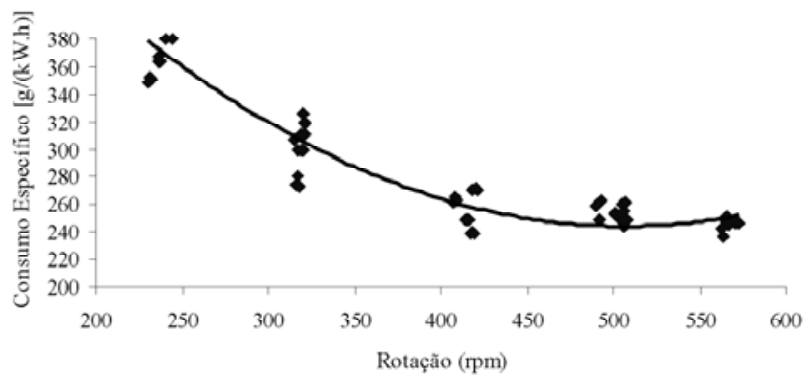

Figura 6 - Curva de tendência do consumo específico para óleo diesel.

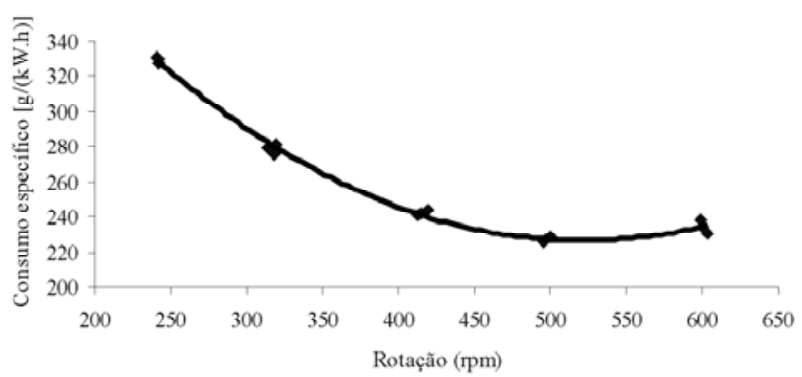

Figura 7 - Curva de tendência do consumo específico para B100 soja.

\section{CONCLUSÃO}

De acordo com as condições operacionais observadas na época da pesquisa, pôde-se obter os seguintes conclusões:

Os testes realizados mostraram a viabilidade de operação de um motor ciclo diesel com biocombustível (B100) soja.

Houve perda de torque com a utilização do biocombustível, sendo $10,7 \%$ menor ao diesel na rotação de trabalho.

O diesel fóssil obteve resultados de potência reduzida melhor que o biodiesel de soja, sendo 6,1\% maior ao biodiesel, na rotação de trabalho.

$\mathrm{O}$ biodiesel de soja apresentou menor consumo específico e energético em relação ao diesel, sendo 14,66 $\%$ menor na rotação de trabalho (540 rpm). 


\section{REFERÊNCIAS BIBLIOGRÁFICAS}

ASSOCIAÇÃO BRASILEIRA DE NORMAS TÉCNICAS. NBR ISO 1585: veículos rodoviários: código de ensaio de motores: potência líquida efetiva. Rio de Janeiro, 1996.

BARBOSA, R. L.; SILVA, F. M. DA; SALVADOR, N.; VOLPATO, C. E. S. Desempenho comparativo de um motor de ciclo diesel utilizando diesel e misturas de biodiesel. Ciência e Agrotecnologia, Lavras, v. 32, n. 5, p. 1588-1593, set./out., 2008.

COSTA NETO, P. R.; ROSSI, L. F. S.; ZANGONEL, G. F.; RAMOS, L. P. Produção de biocombustível alternativo ao óleo diesel através da transesterificação de óleo de soja usado em frituras. Química Nova, São Paulo, v. 23, n. 4, p. 531-537, jul./ago. 2000.

FERRARI, R. A.; SCABIO, A.; OLIVIERA, V. S. Produção e uso de biodiesel etílico na UEPG. Disponível em: <http://uepg.br/propesp/publicatio/exa/2004_6/06.pdf>. Acesso em: 23 abr. 2007.

MAZIERO, J. V. G.; CORRÊA, I. M.; TRIELLI, M. A.; BERNADINI, J. A.; AGOSTINI, M. D'. Avaliação do desempenho de um motor de ignição por compressão utilizando óleo diesel e éster etílico de óleo de girassol como combustível. In: CONGRESSO BRASILEIRO DE PLANTAS OLEAGINOSAS, ÓLEOS VEGETAIS E BIODIESEL, 2., 2005, Varginha. Anais... Lavras: UFLA, 2005. p. 729-733.

MIALHE, L. G. Máquinas motoras na agricultura. São Paulo: Pedagógica e Universitária/EDUSP, 1980. v. 1, 289 p.

MIALHE, L. G. Ensaio \& certificação de máquinas motoras. In: Máquinas agrícolas: ensaios \& certificação. Piracicaba: FEALQ, 1996. cap. 7, p. 321370 .

MOREIRA, A. Combustíveis. São Carlos: USP-Escola de Engenharia de São Carlos, 2007. Apostila. Disponível em: $<$ http://www.netef.eesc.sc.usp.br/moreira/

COMBUSTÍVEIS.ppt>. Acesso em: 28 maio 2007.

OVEG, I. Óleos vegetais: experiência de uso automotivo. Brasília, DF: Ministério da Indústria e do Comércio, Secretaria de Tecnologia Industrial, 1985. 344 p.

RABELO, I. D.; HATAKEYAMA, K.; CRUZ, C. M. S. Estudo de desempenho de combustíveis convencionais associados a biodiesel obtido pela transesterificação de óleo usado em fritura. Disponível em: $<$ www.cefet.br/revistaedução\&tecnologia $>$. Acesso em: 10 jun. 2007.

SALVADOR, N. Desempenho de um motor de ciclo Diesel utilizando o Éster Metílico do óleo de Joannesia Princeps, Vell., em substituição e em misturas com o óleo Diesel. 1984. 54 f. Dissertação (Mestrado em Engenharia Agrícola) - Universidade Federal de Viçosa, Viçosa, 1984.

SILVA, F. M. da; LOPES, A.; CARNEIRO NETO, P.; DABDOUB, M.; SALVADOR, N.; SILVA, R. P. da. Desempenho comparativo de motor de combustão alimentado com diesel, B50 e B100. In: CONGRESSO BRASILEIRO DE PLANTAS OLEAGINOSAS, ÓLEOS, GORDURAS E BIODIESEL, 2004, Varginha. Anais... Lavras: UFLA, 2004. CD-ROM.

TORRES, E. A.; SANTOS, D. C.; SOUZA, D. V. D.; PEIXOTO, L. B.; FRANÇA, T. Ensaio de motores estacionários do ciclo diesel utilizando óleo diesel e biodiesel (B100). [S.1.]: AGRENER, 2006. 